\title{
The inhibitive effect of sh-HIFIA-AS2 on the
}

\section{proliferation, invasion, and pathological damage of breast cancer via targeting miR-548c-3p through regulating HIF-I $\alpha /$ VEGF pathway in vitro and vivo}

This article was published in the following Dove Medical Press journal: OncoTargets and Therapy

\author{
Xiao Guo ${ }^{1, *}$ \\ Shenghai Lee ${ }^{2, *}$ \\ Peilong $\mathrm{CaO}^{3}$ \\ 'Department of Breast Surgery, \\ Central Clinical College of \\ Gynecology Obstetrics, Tianjin \\ Medical University, Tianjin 300II0, \\ China; ${ }^{2}$ Department of Surgery, \\ Zhaoqing Medical College, Zhaoqing, \\ Guangdong 526020, China; \\ ${ }^{3}$ Department of Pathology, The First \\ Affiliated Hospital of Xi'an Jiaotong \\ University, Xi'an, Shanxi 7I006I, \\ China
}

*These authors contributed equally to this work
Correspondence: Peilong Cao

Department of Pathology, The First Affiliated Hospital of Xi'an Jiaotong University, No 277 Yanta West Road, Yanta District, Xi'an, Shanxi Province, China

Tel +86 I76 65036839

Email ypeilongcaoxq@sina.com
Background: Breast cancer (BC) has been the commonest malignant tumor with a low survival rate among woman. Long non-coding RNA hypoxia-inducible factor-1 alpha antisense RNA-2 (HIF1A-AS2) was correlated with various cancers.

Purpose: The study aimed to investigate the roles and related underlying molecular mechanisms of HIF1A-AS2 in BC.

Material and methods: Target relationships were speculated by Targetscan 7.0 and confirmed by dual luciferase reporter assay. Proteins levels were monitored by RT-qPCR, Western blot and immunohistochemistry assays. CCK-8 assay, SA- $\beta$-gal staining and transwell assay were used to detect proliferation, senescence and invasion, respectively. Xenograft nude mice were put into use to evaluate the tumor growth and motility.

Results: The present study exhibited that HIF1A-AS2 and hypoxia-inducible factor-1 alpha (HIF-1 $\alpha$ ) were upregulated while miR-548c-3p was downregulated in MDA-MB-231, MCF-7, ZR-75-1, and BT-549 BC cell lines. Bioinformatics analysis showed HIF1A-AS2 and HIF-1 $\alpha$ were two targets of miR-548c-3p, and the target relationship was further confirmed by dual luciferase reporter assay. Moreover, knockdown of HIF1A-AS2 by shRNA (sh-HIF1A-AS2) markedly elevated miR-548c-3p level, and the enhanced miR-548c-3p noticeably suppressed cell proliferation, invasion, and epithelial-mesenchymal transition, and promoted senescence in vitro. In addition, overexpression of HIF-1 $\alpha$ promoted MCF-7 cell invasion. Intriguingly, low expression of HIF1A-AS2 reduced HIF-1 $\alpha$ level by upregulating the expression of miR$548 \mathrm{c}-3 \mathrm{p}$. Furthermore, experiment in xenograft nude mice has indicated that sh-HIF1A-AS2 inhibited tumor growth and motility by targeting miR-548c-3p through regulating HIF-1 $\alpha$ / vascular endothelial growth factor (VEGF) pathway in vivo.

Conclusion: The inhibitive effect of HIF-1 $\alpha$ /VEGF pathway by sh-HIF1A-AS2 through targeting miR-548c-3p plays crucial regulatory roles in BC. Therefore, designing targeted drugs against HIF1A-AS2 provides a new direction for the treatment of BC.

Keywords: breast cancer, oncogenesis, HIF1A-AS2, miR-548c-3p, HIF-1 $\alpha /$ VEGF, MCF-7

\section{Introduction}

Breast cancer (BC), one of the most common malignant tumors in women, is derived from breast tissue and has become a foremost cause of death among woman. In China, the morbidity of $\mathrm{BC}$ has reached $15 \% \cdot{ }^{1-3}$ Approximately $90 \%$ of BC lethality is attributed to the metastasis and the immunity of current therapeutics. ${ }^{46}$ Despite the widespread application of adjunctive therapies, the survival rates of BC patients remain low. ${ }^{7}$ Recently, immunotherapy has elevated the rehabilitation to some extent. 
Nevertheless, there is still an urgent need to investigate the potential molecular mechanism of $\mathrm{BC}$ to explore more effective therapies for the treatment of $\mathrm{BC}^{8}{ }^{8}$

Numerous studies have demonstrated that long noncoding RNAs (lncRNAs) play a critical role in regulation ${ }^{9,10}$ and tumorigenesis. ${ }^{11-13}$ Hypoxia-inducible factor-1 alpha antisense RNA-2 (HIF1A-AS2), an antisense lncRNA (aslncRNA), was proved to be a natural antisense transcript of hypoxia-inducible factor-1alpha $(\mathrm{HIF}-1 \alpha) .{ }^{14}$ Many studies have indicated that HIF1A-AS2 is associated with a variety of cancers, ${ }^{15}$ such as gastric cancer, ${ }^{16}$ bladder cancer, ${ }^{17}$ ischemic stroke, ${ }^{18}$ and colorectal cancer. ${ }^{19}$ However, the roles and molecular mechanisms of HIF1A-AS2 in BC are still unclear.

Hypoxia is usually witnessed in the tumor environment once the cell growth takes place rapidly. ${ }^{20} \mathrm{HIF}-1 \alpha$, an oxygensensitive transcription factor, enables the transcription of multifarious proangiogenic cytokines such as vascular endothelial growth factor (VEGF). It has been reported that HIF$1 \alpha$-mediated VEGF signaling pathway plays a crucial role in breast tumorigenesis. ${ }^{21}$ Previous studies showed that accumulation of HIF-1 $\alpha$ under ischemic and hypoxic conditions contributed to the inhibitive effect of degradation mediated by ubiquitination. Additionally, a novel evidence was presented that HIF- $1 \alpha$ abundance was controlled by miRNA as well. ${ }^{18,22}$

miR-548c is a member of miR-548 which originated from an inverted repeat transposition element. The mature miR-548c-3p is obtained from miR-548c and consists of 22 nucleotides. A mass of studies have indicated that miR-548c-3p is involved in various cancers, including prostate cancer, ${ }^{23}$ glioma, ${ }^{24}$ gastric cancer, ${ }^{25}$ and BC. ${ }^{26}$ Nevertheless, the underlying molecular mechanisms of miR-548c-3p in BC remains undiscovered.

This study aimed to investigate the roles and related underlying molecular mechanism of HIF1A-AS2 in BC in vivo and vitro. In the present study, we revealed a novel mechanism of HIF1A-AS2 in tumorigenesis of BC, asserting that designing targeted drugs against HIF1A-AS2 provide a new direction for the treatment of $\mathrm{BC}$.

\section{Materials and methods}

\section{Cell lines and cell culture}

Four BC cell lines (MDA-MB-231, MCF-7, ZR-75-1, BT-549) and immortalized normal BC cell line MCF-10A were purchased from the Type Culture Collection of the Chinese Academy of Science. (Shanghai, China). MCF-10A cells were cultured in DMEM (Thermo Fisher Scientific, Waltham, MA, USA) containing $10 \mathrm{mg} / \mathrm{mL} \mathrm{NaHCO}_{3}$. ZR-75-1 cells were cultured in DMEM supplemented with $10 \% \mathrm{FBS}, 0.37 \% \mathrm{NaHCO}_{3}, 10 \mathrm{mM}$ 2-(4-[2-hydroxyethyl]1-piperazinyl) ethanesulfonic acid, $100 \mathrm{U} / \mathrm{mL}$ penicillin, and
$1 \mu \mathrm{g} / \mathrm{mL}$ streptomycin (Thermo Fisher Scientific). MCF-7 and BT-549 cells were cultured in Roswell Park Memorial Institute-1640 medium including $10 \% \mathrm{FBS}, 0.2 \% \mathrm{NaHCO}_{3}$, 10 mM 2-(4-[2-Hydroxyethyl]-1-piperazinyl) ethanesulfonic acid, $100 \mathrm{U} / \mathrm{mL}$ penicillin, and $1 \mu \mathrm{g} / \mathrm{mL}$ streptomycin, pH 7.2 (Thermo Fisher Scientific). MDA-MB-231 cells were cultured in Leibovitz medium (L-15) with 10\% FBS, $100 \mathrm{U} / \mathrm{mL}$ penicillin, and $1 \mu \mathrm{g} / \mathrm{mL}$ streptomycin, $\mathrm{pH} 7.2$ (Thermo Fisher Scientific). All cells were cultivated at $37^{\circ} \mathrm{C}$ with $5 \% \mathrm{CO}_{2}$. To investigate the interaction between HIF1AAS2 and HIF- $1 \alpha$, cells were incubated in an atmosphere containing $1 \% \mathrm{O}_{2}$ and $5 \% \mathrm{CO}_{2}$ at $37^{\circ} \mathrm{C}$.

\section{Cell transfection}

sh-HIF1A-AS2, Ad-HIF1A-AS2, pLenti-CMV-HIF-1 $\alpha$, miR-548c-3p inhibitor, miR-548c-3p mimic, and negative control were provided by Gene Pharma (Shanghai, China). Transfection of MCF-7 cells was conducted using Lipofectamine 2000 transfection reagent (Thermo Fisher Scientific). Cells were cultured in six-well plates for 48 hours and collected for subsequent analyses.

\section{Reverse transcription quantitative real-time PCR (RT-qPCR)}

Total RNAs were extracted from MDA-MB-231, MCF-7, ZR-75-1, BT-549, MCF-10A, and tumor tissues with Trizol reagent (Thermo Fisher Scientific) and reverse-transcribed to cDNA by RevertAid First Strand cDNA Synthesis kit (Thermo Fisher Scientific). RT-qPCR was conducted by SYBR-Green PCR Master Mix kit (Takara, Tokyo, Japan) and ABI 7500 Real-Time PCR System (Thermo Fisher Scientific). All operations were carried out in accordance with the corresponding instructions.

The primers of GAPDH were 5'-GTCAGGAT CCACTCATCACG-3' (sense) and 5'-GATCGGACTTACGG ACTCACATC-3' (antisense). The primers of $\beta$-actin were 5'-TCACCGAGCGCGGCT-3' (sense) and 5'-TAATGTCAC GCACGATTTCCC-3' (antisense). The primers of HIF1AAS2 were 5'-TGATCCAGTCAAAGATTCGCA-3' (sense) and 5'-TCCATGATGCCAGCTCGATCTCACAG-3' (antisense). The primers of miR-548c-3p were $5^{\prime}$-TGTCCTTAG CACCTGGTACGGACCGCT-3' (sense) and 5'-TGCA ATCTGGACTACCGATGG-3' (antisense). The primers of HIF- $1 \alpha$ were 5'-AGTAATCGGACTACCGGAC GTG-3' (sense) and 5'-TGGGCATTACATCGCATG CATC- $3^{\prime}$ (antisense). GAPDH and $\beta$-actin were used as internal references. Fold changes were calculated by the equation $2^{-\Delta \Delta \mathrm{Ct}}$. All experiments were repeated three times. 


\section{Dual luciferase reporter assay}

Targetscan7.0 (http://www.targetscan.org) was used to predict the target of miR-548c-3p on the lncRNA HIF1A-AS2 3'untranslated region (3'UTR). The 3'UTR segments of HIF1A-AS2 (wild type [wt] and mutant [mut]) were amplified and inserted into the luciferase reporter vector (pmirGLO; Promega Corporation, Fitchburg, WI, USA). MCF-7 cells $\left(8 \times 10^{4}\right.$ cells $/$ well $)$ were transfected with Luc-HIF1AAS2-wt, Luc-HIF1A-AS2-mut, or in combination with miRNA-548c using Lipofectamine 2000 transfection reagent (Thermo Fisher Scientific). The luciferase activities were detected by Dual-Luciferase Reporter Assay kit (Promega Corporation). All experiments were repeated three times.

\section{Western blot analysis}

MCF-7 cells and tumor tissues were ground in liquid nitrogen and lysed in lysis buffer (Beyotime, Shanghai, China). After separation by $8 \%$ SDS poly-acrylamide gel, the objective proteins were transferred to polyvinylidene difluoride membranes (IPFL00010; EMD Millipore, Billerica, MA, USA). Primary antibodies specific to Ki-67 (sc-23900, 1:1,000), VEGF (sc-7269, 1:1,000), PCNA (sc71858, 1:1,000), E-cadherin (sc-8426, 1:1,000), vimentin (sc-80975, 1:1,000), N-cadherin (sc-8424, 1:1,000), HIF-1 $\alpha$ (sc-13515, 1:1,000), and GAPDH (sc-66163, 1:1,000) were applied. Then, the samples were incubated with secondary antibodies (sc-516102; Santa Cruz Biotechnology Inc., Dallas, TX, USA) and detected with the enhanced chemiluminescence substrate kit (Amersham Biosciences). The relative protein level was analyzed by using ImageJ software. All experiments were repeated three times.

\section{Cell proliferation}

Proliferative ability of MCF-7 cells was measured by Cell Counting Kit-8 (CCK-8) (Dojindo, Kumamoto, Japan) according to the manufacturer's protocol. MCF-7 cells were transfected with sh-HIF1A-AS2 or miR-548c inhibitor and in combination with miR-548c inhibitor and incubated in $10 \%$ CCK -8 at $37^{\circ} \mathrm{C}$ for 3 hours. Proliferation rates were measured at $0,1,2,3$, and 4 days, respectively. The absorbance was determined at $570 \mathrm{~nm}$ with multifunctional microplate reader SpectraMax M5 (Molecular Devices, Sunnyvale, CA, USA). All experiments were repeated three times.

\section{Cell senescence}

Cell senescence was measured with the senescence-associated $\beta$-galactosidase (SA- $\beta$-gal) staining kit (Beyotime) according to the manufacturer's protocol. All experiments were repeated three times.

\section{Transwell assay}

Transwell assay was conducted to monitor the invasive capacity of tumor cells. Matrigel was coated on the surface of the upper chamber after diluting with serum-free DMEM medium. This was air dried at room temperature for later use. After starving the culture with serum-free medium for 24 hours, the cell suspension was transferred to the apical chambers (200 $\mathrm{mL}$ per chamber). The lower chambers were loaded with $600 \mu \mathrm{L}$ of medium containing $10 \%$ FBS. After incubation at $37^{\circ} \mathrm{C}$ for 48 hours, the chambers were pulled out and washed with PBS. A cotton bud was used to expunge the residual cells on the upper membranes. After fixing with $95 \%$ alcohol and staining with crystal violet, the cells were photographed under a microscope (Leica Microsystems, Wetzlar, Germany). The experiments were independently repeated in triplicate.

\section{Animal model}

All animal experiments were performed in accordance with the National Institutes of Health Guide for the Care and Use of Laboratory Animals and were approved by the Central Clinical College of Gynecology Obstetrics of Tianjin Medical University. A total of $60 \mathrm{BALB} / \mathrm{c}$ nude mice (male, 4-week-old) were obtained from the Animal Center of Tianjin Medical University and housed in a controlled environment at $25^{\circ} \mathrm{C} \pm 3^{\circ} \mathrm{C}$ and $60 \%$ humidity, in a 12-hour light/dark cycle with free access to food and water. Untreated MCF-7 cells or MCF-7 cells $\left(2 \times 10^{5}\right)$ transfected with sh-HIF1A-AS2 were subcutaneously injected into the flank area of mice to form tumors. After successful modeling, mice were divided into two groups with ten in each group (repeat three times): control group and sh-HIF1AAS2 group (mice injected with MCF-7 cells transfected with sh-HIF1A-AS2). Tumor weight was measured at 25 days postinjection. After 25 days, rats were euthanized by intraperitoneal injection of pentobarbital sodium ( $200 \mathrm{mg} / \mathrm{kg}$ body weight). Tumors were collected for the following experiments.

\section{Immunohistochemistry (IHC)}

IHC analysis was performed according to a previously described method. ${ }^{27}$ Briefly, $5 \mu \mathrm{m}$ thick paraffin sections were deparaffinized in xylene and rehydrated in ethanol at different gradients. After that, the tissue slices were incubated in 30\% $\mathrm{H}_{2} \mathrm{O}_{2}$ for 30 minutes to inactivate endogenous peroxidase. After antigen retrieval in $10 \mathrm{mM}$ heated citrate buffer for 10 minutes, the tissue sections were incubated with primary antibodies Ki-67 (\#9027, 1:400; CST, St Louis, MO, USA) and VEGF ( $\# 9698,1: 1600$; CST) overnight at $4^{\circ} \mathrm{C}$. Corresponding secondary antibody (\#8114; CST) was added and incubated for 1 hour at room temperature. The images were observed under a microscope (E200; Nikon, Tokyo, Japan). 


\section{Statistical analysis}

The statistical analysis was conducted with SPSS 21.0 (SPSS, Inc, Chicago, IL, USA). Measurement data were presented as mean $\pm \mathrm{SD}(\mathrm{x} \pm \mathrm{s})$, and data consistent with the normal distribution were analyzed by independent $t$-test. Multiple sets of data were analyzed by one-way ANOVA followed by Bonferroni post hoc test. Enumeration data were presented as a percentage or ratio and verified with chi-squared test. $P<0.05$ was considered statistically significant.

\section{Results}

\section{Increase of HIFIA-AS2 and decrease of miR-548c-3p in human BC cells}

RT-qPCR was conducted to monitor the expression levels of HIF1A-AS2 and miR-548c-3p. As shown in Figure 1A, HIF1A-AS2 was significantly increased in four BC cell lines (MDA-MB-231, MCF-7, ZR-75-1, and BT-549) compared with normal mammary epithelial cell line (MCF-10A) $(P<0.05, P<0.01)$, whereas miR-548c-3p was significantly decreased (Figure 1B; $P<0.05, P<0.01$ ). MCF-7 cell line was used as the follow-up experimental cell line due to the high significant differences observed with MCF-10A $(P<0.01)$. Taken together, these results demonstrated that HIF1A-AS2 and miR-548c-3p might be correlated with human BC with contrary effects.

\section{miR-548c-3p targeted HIFIA-AS2 and was negatively regulated by HIFIA-AS2 in MCF-7 cells}

Targetscan7.0 (http://www.targetscan.org) was used to predict the presumptive target of miR-548c-3p on HIF1A-AS2
3'UTR (wt and mut) (Figure 2A). miR-548c-3p was measured by RT-qPCR. As shown in Figure 2B, miR-548c-3p was noticeably upregulated in MCF-7 cells transfected with sh-HIF1A-AS2. A reversed result was observed while transfected with miR-548c-3p inhibitor (Figure 2B; $P<0.05$ ). Besides, overexpression of HIF1A-AS2 significantly inhibited the expression of miR-548c-3p (Figure 2C; $P<0.05$ ), whereas a reversed result was observed in MCF-7 cells transfected with miR-548c-3p mimic (Figure 2C; $P<0.001$ ). Furthermore, luciferase reporter assay further confirmed the correlation between HIF1A-AS2 and miR-548c-3p. The results of the luciferase reporter assay clearly showed a dramatic downregulation in luciferase activity (Figure 2D; $P<0.05$ ), while no marked effect was observed with the transfection of HIF1A-AS2 mut and miR-548c mimic. These results demonstrated that miR-548c-3p targets HIF1A-AS2 $3^{\prime}$ UTR leading to the downregulation of luciferase activity (Figure 2D; $P>0.05$ ). In short, these results indicated that HIF1A-AS2 negatively regulated miR-548c-3p in MCF-7 cells.

\section{sh-HIFI A-AS2 suppressed proliferation and senescence by regulating miR-548c- $3 p$ in MCF-7 cells}

CCK-8 assay was performed to investigate proliferation, while SA- $\beta$-gal staining was applied to investigate senescence and Western blot was used for further verification of cell proliferative potential. As shown in Figure 3A, results showed that proliferation capacity was significantly impeded in MCF-7 cells after transfection with sh-HIF1A-AS2 (Figure 3A; $P<0.05, P<0.01$ ). Meanwhile, Western blot
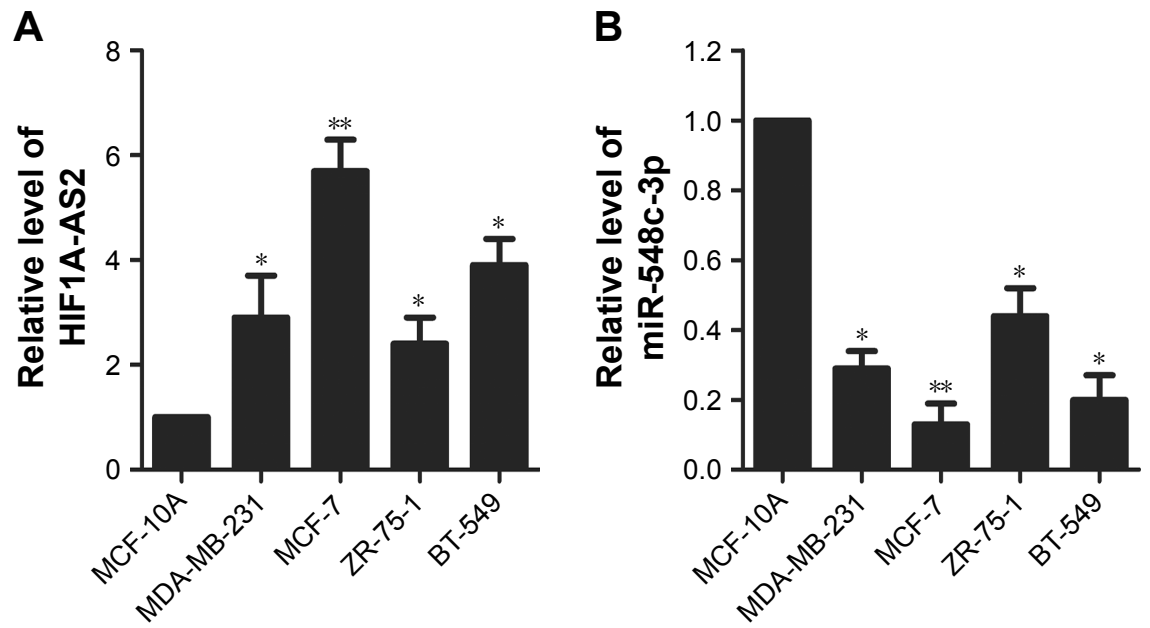

Figure I Increase of HIFIA-AS2 and decrease of miR-548c-3p in human BC cells.

Notes: (A) The expression level of HIFIA-AS2 was measured by RT-qPCR in MCF-IOA and BC cell lines (MDA-MB-23I, MCF-7, ZR-75-I, and BT-549). (B) The expression level of miR-548c-3p was detected by RT-qPCR in MCF-IOA and BC cell lines (MDA-MB-23I, MCF-7, ZR-75-I, and BT-549). *P <0.05 vs control; **P $<0.0$ I vs control. Abbreviations: BC, breast cancer; HIFIA-AS2, hypoxia-inducible factor-I alpha antisense RNA-2; RT-qPCR, quantitative real-time PCR. 

A HIF1A-AS2 mut
5'...GGATTCATAGTGACCCCCCTGTTTTA...3'
HIF1A-AS2 wt
5'...GGATTCATAGTGATTTTTCTGTTTTA...3'
hsa-miR-548c-3p 3' CGUUUUCAUUAACUCUAAAAAC 5'

B

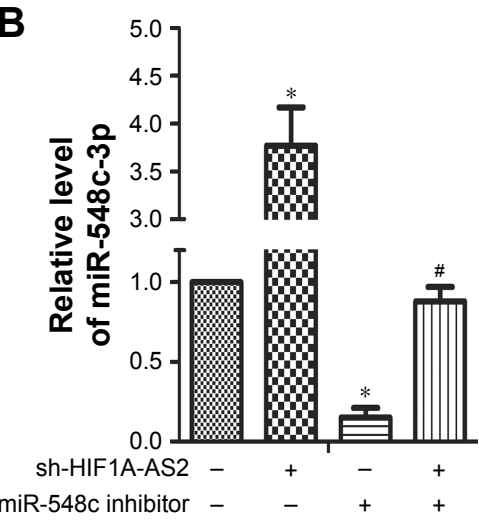

C

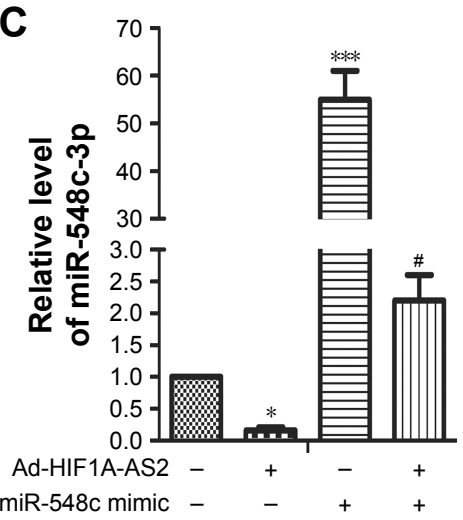

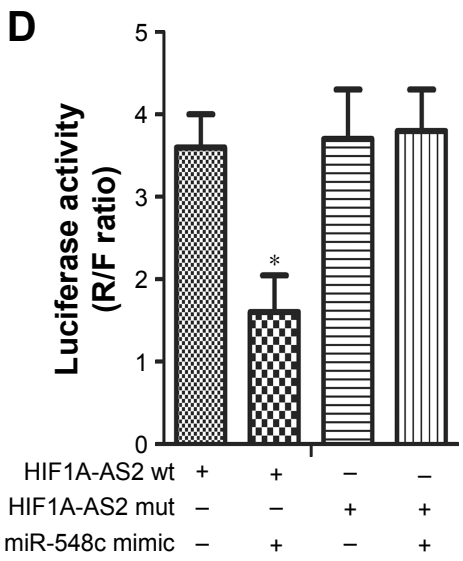

Figure 2 miR-548c-3p targeted HIFIA-AS2 and was negatively regulated by HIFIA-AS2 in MCF-7 cells

Notes: (A) The target of miR-548c-3p on HIFIA-AS2 3'UTR (wt and mut). (B) The expression level of miR-548c-3p was detected by RT-qPCR in MCF-7 cells transfected with sh-HIFIA-AS2 or miR-548c inhibitor and in combination with miR-548c inhibitor ( ${ }^{*} P<0.05$ vs control, ${ }^{*} P<0.05$ vs miR-548c inhibitor group). (C) The expression level of miR$548 c-3 p$ was detected by RT-qPCR in MCF-7 cells transfected with Ad-HIFIA-AS2 or miR-548c mimic and in combination with miR-548c mimic $(* P<0.05$ vs control, $* * * P<0.00$ I vs control, $\#$ P $<0.05$ vs miR-548c mimic group). (D) The activity of HIFIA-AS2 was measured by dual luciferase reporter assay in MCF-7 cells co-transfected with HIFIA-AS2 (wt and mut) and miR-548c mimics ( $* P<0.05$ vs control). "R" represents the relative light unit of Ranilla luciferase. "F" represents the relative light unit of Firefly luciferase.

Abbreviations: HIFIA-AS2, hypoxia-inducible factor-I alpha antisense RNA-2; mut, mutant; RT-qPCR, quantitative real-time PCR; UTR, untranslated region; wt, wild type.

assays exhibited that the proliferation marker proteins levels (Ki-67 and PCNA) were downregulated in MCF-7 cells after transfection with sh-HIF1A-AS2, and the inhibition was counteracted by miR-548c-3p inhibitor (Figure $3 \mathrm{C}$ and D; $P<0.05)$. Besides, SA- $\beta$-gal staining indicated that cell senescence was enhanced in MCF-7 cells transfected with sh-HIF1A-AS2 (Figure 3B; $P<0.01$ ). Taken together, our results indicated that sh-HIF1A-AS2 played an important role in MCF-7 cell proliferation and senescence by regulating miR-548c-3p.

\section{sh-HIF I A-AS2 inhibited cell invasion and EMT by regulating miR-548c-3p in MCF-7 cells}

Transwell assay and Western blot were applied to investigate the invasive capacity and EMT process of MCF-7 cells,
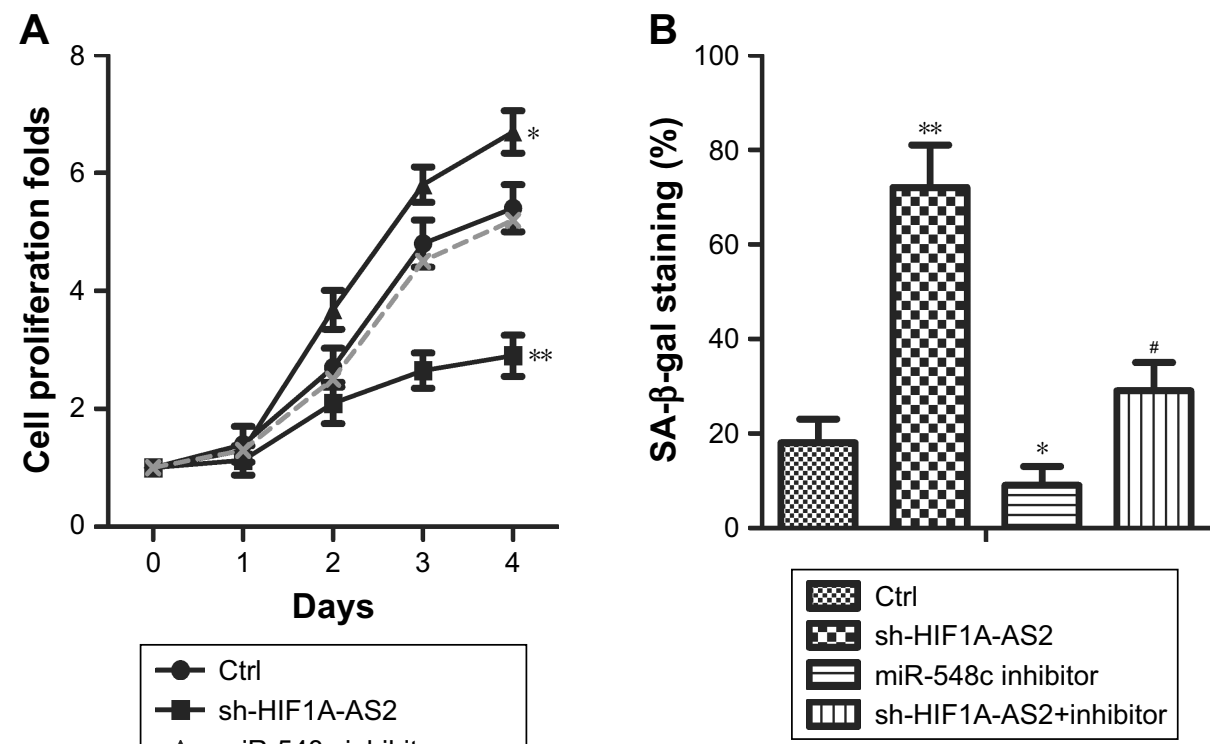

Figure 3 (Continued) 

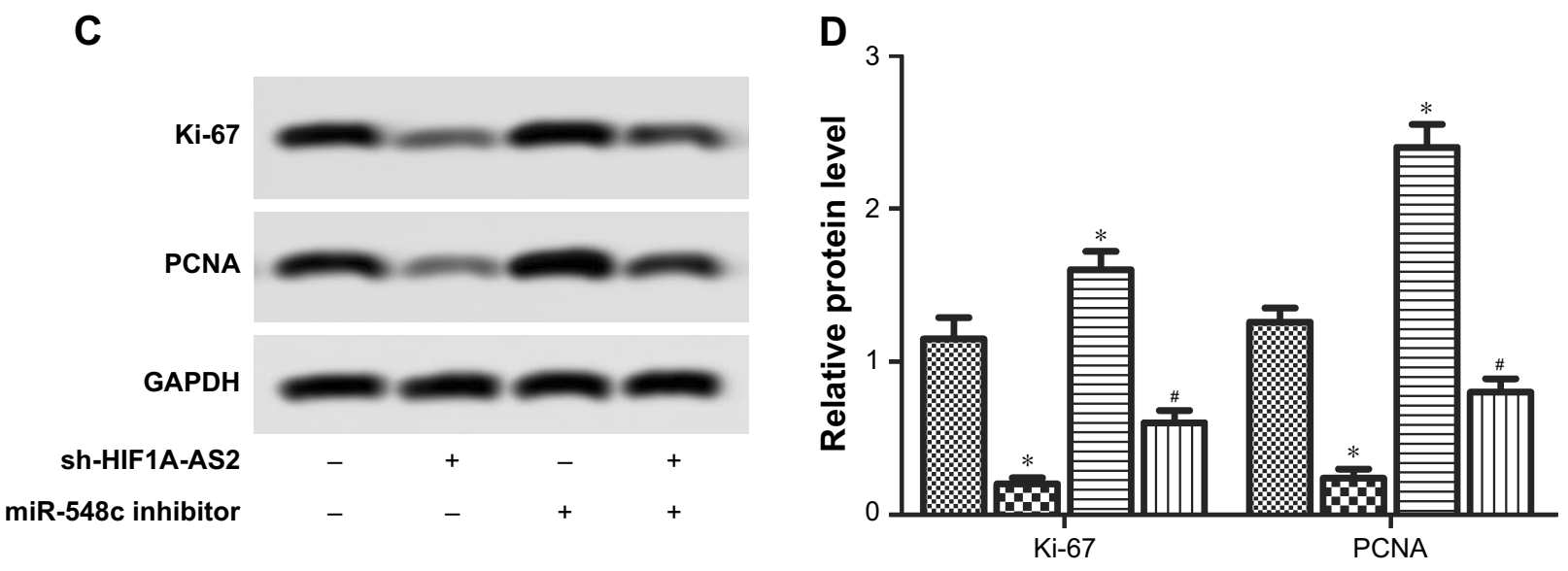

Figure 3 sh-HIFIA-AS2 suppressed proliferation and senescence by regulating miR-548c-3p in MCF-7 cells.

Notes: (A) The cell proliferation folds were detected by CCK-8 in MCF-7 cells transfected with sh-HIFIA-AS2 or miR-548c inhibitor and in combination with miR-548c inhibitor $\left(* P<0.05\right.$ vs control, ${ }^{* *} P<0.01$ vs control). (B) The cell senescence was detected by SA- $\beta$-gal staining in MCF-7 cells transfected with sh-HIFIA-AS2 or miR-548c inhibitor and in combination with miR-548c inhibitor ( $* P<0.05$ vs control, ${ }^{* * P}<0.0$ I vs control, ${ }^{*} P<0.05$ vs miR-548c inhibitor group). (C) The expression of proliferation marker proteins Ki-67 and PCNA were measured by Western blot in MCF-7 cells transfected with sh-HIFIA-AS2 or miR-548c inhibitor and in combination with miR-548c inhibitor. (D) Histograms display the statistical analysis of Western blotting results. The bars show mean \pm SD of three independent experiments ( ${ }^{*} P<0.05$ vs control, $\# P<0.05$ vs miR-548c inhibitor group). Abbreviations: CCK-8, Cell Counting Kit-8; Ctrl, control; HIFIA-AS2, hypoxia-inducible factor-I alpha antisense RNA-2; SA- $\beta$-gal, senescence-associated $\beta$-galactosidase.

respectively. As shown in Figure 4A, the downregulation of HIF1A-AS2 significantly impeded MCF-7 cell invasion. After transfection with miR-548c-3p inhibitor, the number of invasive cells was significantly increased (Figure $4 \mathrm{~A}, P<0.05$ ).
Generally, increase of E-cadherin and decrease of vimentin/Ncadherin expression are the fundamental events in EMT. As shown in Figure 4B, low expression of HIF1A-AS2 observably increased E-cadherin protein level and decreased the
A

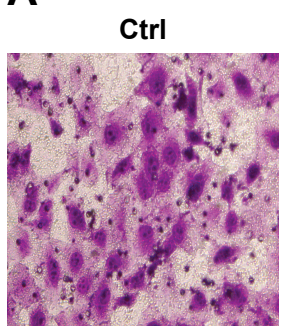

sh-HIF1A-AS2

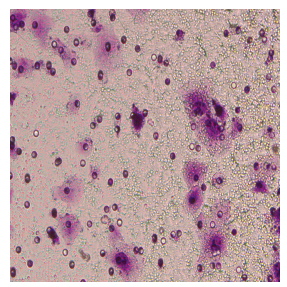

miR-548c inhibitor

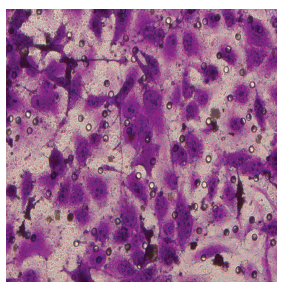

sh-HIF1A-AS2+ inhibitor

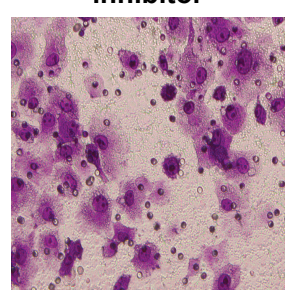

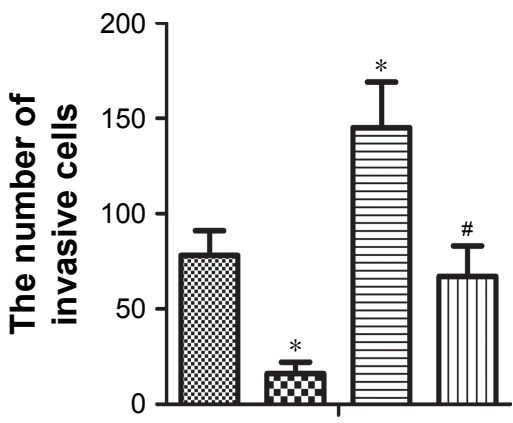

B
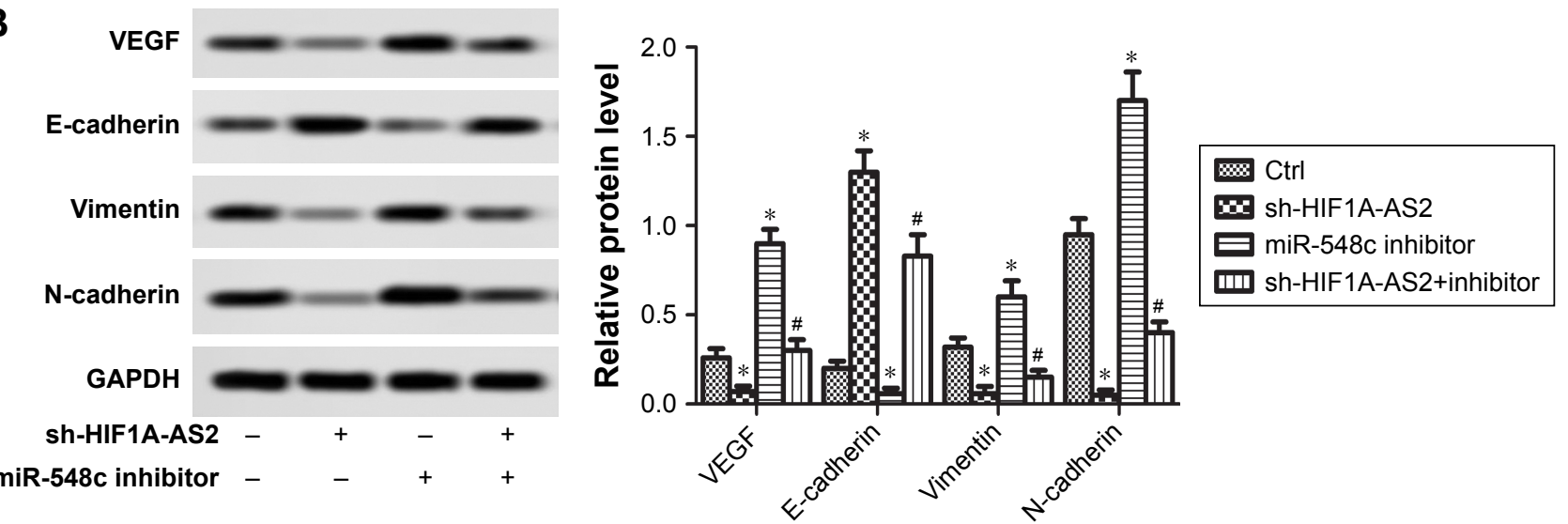

Figure 4 sh-HIFIA-AS2 inhibited cell invasion and epithelial-mesenchymal transition (EMT) by regulating miR-548c-3p in MCF-7 cells.

Notes: (A) The invasion ability of MCF-7 cells transfected with sh-HIFIA-AS2 or miR-548c inhibitor and in combination with miR-548c inhibitor was detected by transwell assays. Invasive cells were stained with crystal violet solution, and the quantification of invasive cells is shown ( $* P<0.05$ vs control, $\# P<0.05$ vs miR-548c inhibitor group). (B) The expression of EMT marker proteins and VEGF was measured by Western blot in MCF-7 cells transfected with sh-HIFIA-AS2 or miR-548c inhibitor and in combination with miR-548c inhibitor ( $* P<0.05$ vs control, ${ }^{*} P<0.05$ vs miR-548c inhibitor group).

Abbreviations: Ctrl, control; HIFIA-AS2, hypoxia-inducible factor-I alpha antisense RNA-2; VEGF, vascular endothelial growth factor. 
levels of vimentin and $\mathrm{N}$-cadherin $(P<0.05)$, whereas the miR-548c-3p inhibitor showed opposite effects $(P<0.05)$. Additionally, the expression of VEGF was significantly restrained in MCF-7 cells with the transfection of sh-HIF1AAS2. Therefore, our research declared that sh-HIF1A-AS2 restrained cell motility through the inhibitive effect of EMT process via regulating miR-548c-3p in MCF-7 cells.

\section{sh-HIFIA-AS2 inhibited cell motility via regulating HIF-I $\alpha /$ VEGF signaling pathway in MCF-7 cells}

To investigate the potential molecular mechanism of sh-HIF1A-AS2 on BC cells, Targetscan7.0 (http://www. targetscan.org), Western blot, and transwell assay were performed. The presumptive target of HIF-1 $\alpha$ was shown to be present on miR-548c-3p 3'UTR (wt and mut) (Figure 5A). In addition, the four BC cell lines (MDA-MB-231, MCF-7, ZR-75-1, and BT-549) and MCF-10A were incubated under $1 \% \mathrm{O}_{2}$ for 48 hours, and HIF- $1 \alpha$ was measured by RT-qPCR. As shown in Figure 5B, HIF-1 $\alpha$ was noticeably upregulated in the four BC cell lines compared with MCF-10A $(P<0.05$,
$P<0.01)$. Western blot results showed that levels of mesenchymal marker proteins (vimentin), HIF-1 $\alpha$, and VEGF were markedly suppressed in MCF-7 cells transfected with sh-HIF1A-AS2 (Figure 5C, $P<0.05$ ). However, a reversed result was observed in $\mathrm{MCF}-7$ cells transfected with pLentiCMV-HIF- $1 \alpha$ to overexpress HIF- $1 \alpha$ (Figure 5 C; $P<0.05$ ). Besides, knockdown of HIF1A-AS2 by shRNA significantly inhibited invasion, whereas overexpression of HIF-1 $\alpha$ had opposite results (Figure 5D and E; $P<0.05$ ). All these data suggested that sh-HIF1A-AS2 inhibited cell motility via regulating HIF-1 $\alpha$ /VEGF signaling pathway in MCF-7 cells.

\section{sh-HIFI A-AS2 inhibited tumor growth and motility by targeting miR-548c-3p to regulate HIF-I $\alpha /$ VEGF pathway in vivo}

The in-situ tumor was taken out and weighed 25 days later. As shown in Figure 6A, the tumor weight was noticeably reduced in xenograft nude mice injected with MCF-7 cells with sh-HIF1A-AS2 $(P<0.01)$, indicating that tumor growth was markedly inhibited in sh-HIF1A-AS2 mice. In addition, RT-qPCR results showed that after subcutaneous injection

\section{A Position 1,553-1,559 of HIF1A 3'UTR $\quad 5^{\prime}$... UAGAAAAAGAAACAUC-AUUUUUAA... hsa-miR-548c-3p $\quad 3^{\prime} \quad$ CGUUUUCAUUAACUCUAAAAAC}
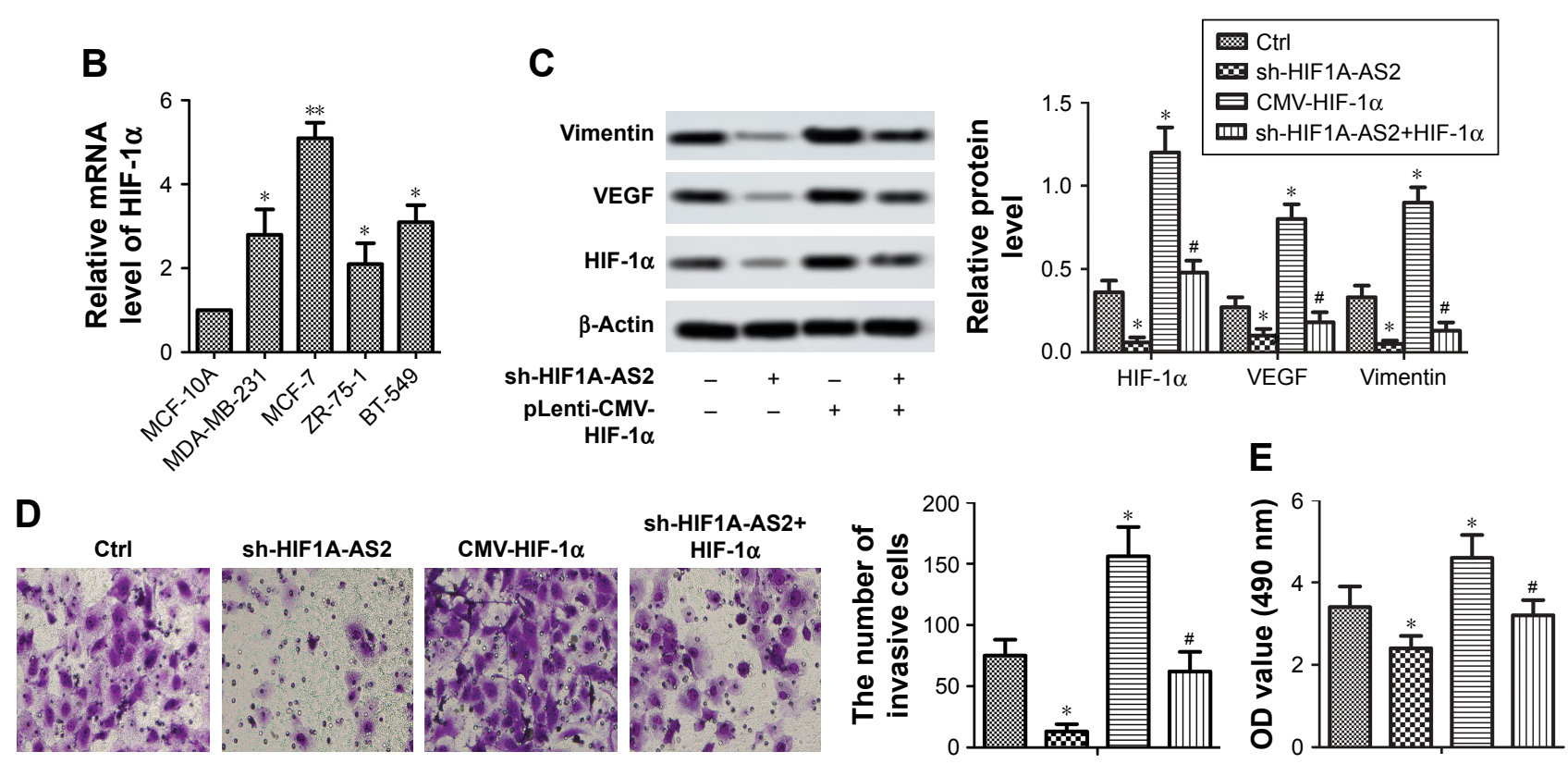

Figure 5 sh-HIFIA-AS2 inhibited cell motility via regulating HIF-I $\alpha /$ VEGF signaling pathway in MCF-7 cells.

Notes: MCF-IOA and BC cells lines (MDA-MB-23I, MCF-7, ZR-75-I, and BT-549) were incubated under I\% $\mathrm{O}_{2}$ for 48 hours. (A) The target of miR-548c-3p on the HIFIA $3^{\prime}$ UTR. (B) The expression level of HIF-I $\alpha$ was detected by RT-qPCR ( $* P<0.05$ vs control; $* * P<0.0$ I vs control). (C) Expression of EMT marker proteins, VEGF, and HIF-I $\alpha$ was measured by Western blot in MCF-7 cells transfected with sh-HIFIA-AS2 or pLenti-CMV-HIF-I $\alpha$ and in combination with pLenti-CMV-HIF-I $\alpha$ ( $* P<0.05$ vs control, ${ }^{\#} P<0.05$ vs CMV-HIF-I $\alpha$ group). (D) Invasion ability of MCF-7 cells transfected with sh-HIFIA-AS2 or pLenti-CMV-HIF-I $\alpha$ and in combination with PLenti-CMV-HIF-I $\alpha$. Invasive cells were stained with crystal violet solution, and the quantification of invasive cells is shown $\left(* P<0.05\right.$ vs control, ${ }^{*} P<0.05$ vs $C M V-H I F-I ~ \alpha$ group). Scale bar $=20 \mu \mathrm{m}$. (E) Proliferation ability was measured by CCK-8 assays in MCF-7 cells transfected with sh-HIFIA-AS2 or pLenti-CMV-HIF-I $\alpha$ and in combination with pLenti-CMV-HIF-I $\alpha$ ( $* \mathrm{P}<0.05$ vs control, ${ }^{*} P<0.05$ vs CMV-HIF- $1 \alpha$ group).

Abbreviations: BC, breast cancer; CCK-8, Cell Counting Kit-8; Ctrl, control; EMT, epithelial -mesenchymal transition; HIFIA-AS2, hypoxia-inducible factor-I alpha antisense RNA-2; RT-qPCR, quantitative real-time PCR; HIF-I $\alpha$, hypoxia-inducible factor-I alpha; UTR, untranslated region; VEGF, vascular endothelial growth factor. 
A

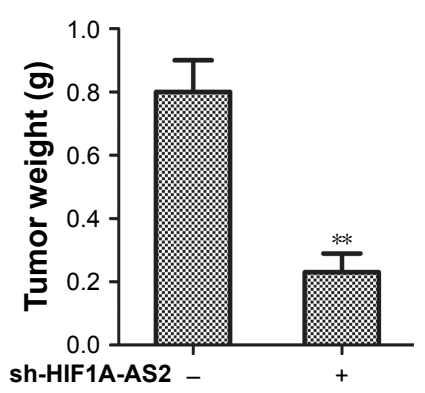

sh-HIF1A-AS2 -
B

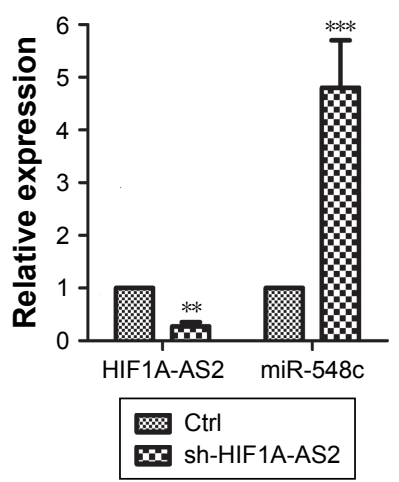

Sample 2

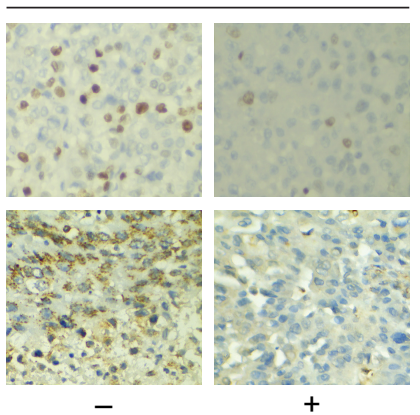

C
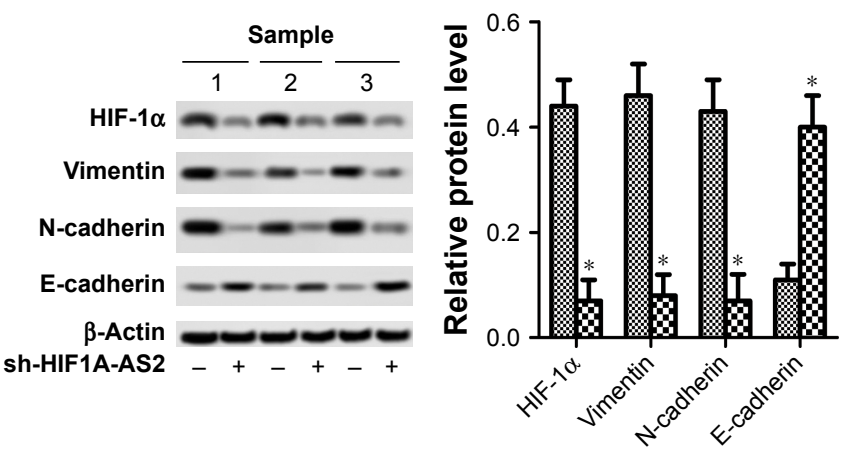

Sample 3
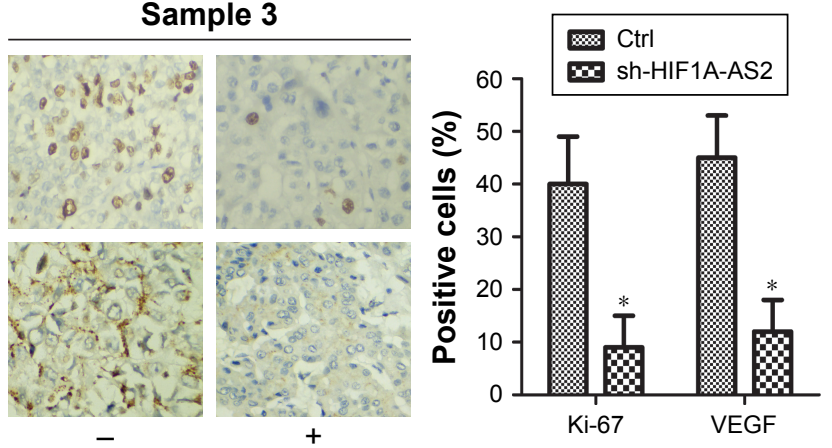

Figure 6 sh-HIFIA-AS2 inhibited tumor growth and motility by targeting miR-548c-3p to regulate HIF-I $\alpha /$ VEGF pathway in vivo.

Notes: (A) Tumor weight was noticeably reduced in sh-HIFIA-AS2 group mice ( $* * P<0.01$ vs control). (B) Expression level of miR-548c-3p was detected by RT-qPCR in shHIFIA-AS2 group mice ( $* * P<0.0$ I vs control; $* * * P<0.00$ I vs control). (C) Expression of EMT marker proteins and HIF-I $\alpha$ was measured by Western blot in sh-HIFIA-AS2 group mice $(* P<0.05$ vs control). (D) Expression of proliferation marker proteins Ki-67 and VEGF in formalin-fixed, paraffin-embedded tumors from sh-HIFIA-AS2 group mice was detected by IHC analysis ( $* P<0.05$ vs control). "-_" stands for Ctrl group; "+" stands for sh-HIFIA-AS2 group.

Abbreviations: Ctrl, control; EMT, epithelial -mesenchymal transition; HIF-I $\alpha$, hypoxia-inducible factor-I alpha; HIFIA-AS2, hypoxia-inducible factor-I alpha antisense RNA-2; IHC, immunohistochemistry; RT-qPCR, quantitative real-time PCR; VEGF, vascular endothelial growth factor.

of MCF-7 cells with sh-HIF1A-AS2, a large amount of miR-548c-3p was accumulated in xenograft nude mice (Figure 6B; $P<0.001$ ). In addition, expression of EMT marker proteins and HIF-1 $\alpha$ was measured by Western blot in sh-HIF1A-AS2 group mice. As shown in Figure 6C, knockdown of HIF1A-AS2 by shRNA significantly inhibited the expression of mesenchymal marker proteins (vimentin and $\mathrm{N}$-cadherin), and promoted the expression of epithelial marker protein (E-cadherin) $(P<0.05)$. Furthermore, IHC analysis results demonstrated that the expression of Ki-67 and VEGF was reduced in formalin-fixed, paraffin-embedded tumors from sh-HIF1A-AS2 group mice (Figure 6D; $P<0.05)$. Thus, we concluded that sh-HIF1A-AS2 inhibited tumor growth and motility by targeting miR-548c-3p to regulate HIF-1 $\alpha$ /VEGF pathway in vivo.

\section{Discussion}

As a delegate of the most common cancer among woman, $\mathrm{BC}$ has been increasing in morbidity worldwide. ${ }^{27,28}$ Although various therapies are available, the survival rates of $\mathrm{BC}$ patients remain low. ${ }^{7}$ Additionally, chemoresistance and adverse reactions extremely impeded the therapeutic effect of BC treatment. ${ }^{29,30} \mathrm{Up}$ to now, no treatment that is completely effective is available. The abnormally expressed proteins that were associated with increased proliferation and motility of cells were regarded as culprits in the development of BC..$^{31,32}$ Therefore, analyzing the underlying molecular mechanism is the most intuitive way for the treatment of BC.

As a hypoxia-dependent lncRNA, HIF1A-AS2 has been reported to be correlated with a variety of cancers. It was first found to be abnormally expressed in nonpapillary clear cell renal carcinomas. ${ }^{33}$ Subsequently, HIF1A-AS2 was proved to be involved in the progression, such as proliferation, migration, and apoptosis of various cancers. Chen et al revealed that overexpression of HIF1A-AS2 promoted cell proliferation, tumor invasion, and lymph node metastasis in gastric cancer ${ }^{16}$ and bladder cancer. ${ }^{17}$ With the deepening of research, Li et al reported that HIF1A-AS2 accelerated cell viability, migration, and tube formation by activating HIF-1 $\alpha /$ VEGF/Notch 1 cascade pathways in human umbilical vein endothelial 
cells. ${ }^{18}$ Besides, HIF1A-AS2 also exerted carcinogenicity in colorectal cancer, thus demonstrating that HIF1A-AS2 might be used as a therapeutic target in colorectal cancer. ${ }^{19}$ Similarly in our study, we found that HIF1A-AS2 played an analogous role in $\mathrm{BC}$ and that sh-HIF1A-AS2 remarkably impeded proliferation, invasion, and EMT in vitro and vivo. miR-548c-3p is a pivotal small RNA molecule involved in cell proliferation, invasion, and tumorigenesis. Studies have shown that miR-548c-3p is widely present in human embryonic stem cells and unfractionated castration-resistant prostate cancer. ${ }^{23}$ Luo et al elucidated that overexpression of miR-548c-3p remarkably suppressed cell viability and promoted apoptosis and G2/M cell cycle arrest. ${ }^{33}$ In addition, miR-548c-3p was also reported to be involved in the inhibitive effect of glioma tissue cell proliferation and migration in vitro. ${ }^{29}$ According to the report of Tormo et al, miR-548c-3p suppressed doxorubicin-treated MCF-7 cell viability in BC. ${ }^{26}$ Consistent with these results, in our study, we manifested that overexpression of HIF1A-AS2 significantly inhibited the expression of miR-548c-3p, while knockdown of HIF1A-AS2 markedly elevated miR-548c-3p level. The enhanced miR$548 \mathrm{c}-3 \mathrm{p}$ levels restrained proliferation, invasion, and EMT, and promoted cell senescence in MCF-7 cells and tumor tissue.

As an oxygen-sensitive transcription factor, HIF-1 $\alpha$ activates transcription of various proangiogenic cytokines such as VEGF. Studies have shown the critical roles of HIF-1 $\alpha$ / VEGF pathway in angiogenesis ${ }^{21,34,35}$ and tumorigenesis. ${ }^{35}$ Previous studies demonstrated that the breast tumor proliferation was inhibited by simvastatin via targeting the AMPK/HIF-1 $\alpha /$ VEGF signaling pathway. ${ }^{34}$ Besides, research by Kim and Ma also suggested that rhaponticin markedly suppressed the angiogenic and metastatic activities by targeting the HIF-1 $\alpha$ /VEGF signaling pathway. ${ }^{36}$ In addition, a novel signaling pathway mTOR/HIF-1 $\alpha /$ VEGF involved in the inhibitive effect of glioma cell proliferation and invasion was found by Nan et al. ${ }^{37}$ Moreover, the expression of HIF-1 $\alpha$ also lead to increased VEGF-A and activation of HIF-1 $\alpha /$ VEGF signaling pathway in lung cancer cells. ${ }^{38}$ Similarly, in our research, we first demonstrated that sh-HIF1A-AS2 represses HIF-1 $\alpha$ /VEGF signaling pathway to impede the $\mathrm{BC}$ cell proliferation, senescence, invasion, and angiogenesis by targeting miR-548c-3p in vitro and vivo.

\section{Conclusion}

In conclusion, our research revealed a novel mechanism of HIF1A-AS2 action in tumorigenesis of BC. The primary mechanisms can be summarized as following: 1) HIF1A-AS2 was upregulated in BC cells and tumor, while
miR-548c-3p was downregulated. In addition, sh-HIF1AAS2 noticeably enhanced the expression of miR-548c-3p in cells and tumor, that is, HIF1A-AS2 has a negative regulation on miR-548c-3p. 2) sh-HIF1A-AS2 noticeably reduced the cell proliferation, invasion, and angiogenesis in cells and tumor. 3) sh-HIF1A-AS2 interacted with miR-548c-3p to inhibit the tumor growth and motility via interfering the HIF-1 $\alpha$ /VEGF pathway in vitro and vivo.

\section{Acknowledgment}

Xiao Guo and Shenghai Lee are co-first authors for this study.

\section{Author contributions}

$\mathrm{XG}, \mathrm{SL}$, and PC conceived and designed this study, were involved in the animal assay and statistical analysis, carried out the cell line-based research, wrote the manuscript, and revised the manuscript. PC provided useful input for the analyses and helped in editing the manuscript. All authors read and approved the final manuscript and agree to be accountable for all aspects of the work in ensuring that questions related to the accuracy or integrity of any part of the work are appropriately investigated and resolved.

\section{Disclosure}

The authors report no conflicts of interest in this work.

\section{References}

1. Torre L, Bray F, Siegel R, et al. Global cancer statistics, 2012. $C A$ Cancer J Clin. 2015;65(2):87-108.

2. Jaffee EM, Dang CV, Agus DB, et al. Future cancer research priorities in the USA: a Lancet oncology Commission. Lancet Oncol. 2017;18(11): e653-e706.

3. Chen W, Zheng R, Baade PD, et al. Cancer statistics in China, 2015. CA Cancer J Clin. 2016;66(2):115-132.

4. Sosa MS, Bragado P, Aguirre-Ghiso JA. Mechanisms of disseminated cancer cell dormancy: an awakening field. Nat Rev Cancer. 2014;14(9): 611-622.

5. Pantel K, Brakenhoff RH. Dissecting the metastatic cascade. Nat Rev Cancer. 2004;4(6):448-456.

6. Gonzalez-Angulo AM, Morales-Vasquez F, Hortobagyi GN. Overview of resistance to systemic therapy in patients with breast cancer. $A d v$ Exp Med Biol. 2007;608(1):1.

7. Kolahdooz F, Jang SL, Corriveau A, Gotay C, Johnston N, Sharma S Knowledge, attitudes, and behaviours towards cancer screening in Indigenous populations: a systematic review. Lancet Oncol. 2014;15(11): e504-e516.

8. Pusztai L, Karn T, Safonov A, Abu-Khalaf MM, Bianchini G. New strategies in breast cancer: immunotherapy. Clin Cancer Res. 2016;22(9): 2105-2110.

9. Guttman M, Rinn JL. Modular regulatory principles of large non-coding RNAs. Nature. 2012;482(7385):339-346.

10. Khalil M, Guttman M, Huarte M, et al. Many human large intergenic noncoding RNAs associate with chromatin-modifying complexes and affect gene expression. Proc Natl Acad Sci USA. 2009;106(28):11667-11672.

11. Yu G, Yao W, Gumireddy K, et al. Pseudogene PTENP1 functions as a competing endogenous RNA to suppress clear-cell renal cell carcinoma progression. Mol Cancer Ther. 2014;13(12):3086-3097. 
12. Yan Y, Han J, Li Z, Yang H, Sui Y, Wang M. Elevated RNA expression of long non-coding HOTAIR promotes cell proliferation and predicts a poor prognosis in patients with diffuse large B cell lymphoma. Mol Med Rep. 2016;13(6):5125-5131.

13. Ying L, Chen Q, Wang Y, Zhou Z, Huang Y, Qiu F. Upregulated MALAT-1 contributes to bladder cancer cell migration by inducing epithelial-to-mesenchymal transition. Mol Biosyst. 2012;8(9):2289-2294.

14. Thrash-Bingham CA, Tartof KD. A HIF: a natural antisense transcript overexpressed in human renal cancer and during hypoxia. J Natl Cancer Inst. 1999;91(2):143-151.

15. Bertozzi D, Iurlaro R, Sordet O, Marinello J, Zaffaroni N, Capranico G. Characterization of novel antisense HIF- $1 \alpha$ transcripts in human cancers. Cell Cycle. 2011;10(18):3189-3197.

16. Chen WM, Huang MD, Kong R, et al. Antisense long noncoding RNA HIF1A-AS2 is upregulated in gastric cancer and associated with poor prognosis. Dig Dis Sci. 2015;60(6):1655-1662.

17. Chen M, Zhuang C, Liu Y, et al. Tetracycline-inducible shRNA targeting antisense long non-coding RNA HIF1A-AS2 represses the malignant phenotypes of bladder cancer. Cancer Lett. 2016;376(1):155-164.

18. Li L, Wang M, Mei Z, et al. IncRNAs HIF1A-AS2 facilitates the upregulation of HIF- $1 \alpha$ by sponging to miR-153-3p, whereby promoting angiogenesis in HUVECs in hypoxia. Biomed Pharmacother. 2017;96: $165-172$.

19. Lin J, Shi Z, Yu Z, He Z. LncRNA HIF1A-AS2 positively affects the progression and EMT formation of colorectal cancer through regulating miR-129-5p and Dnmt3a. Biomed Pharmacother. 2018;98:433-439.

20. Cotran R, Kumar V, Robbins S. Robbins and Cotran Pathologic Basis of Disease [e-book]. 9th edition. 2010.

21. Wang J, Li G, Wang Y, et al. Suppression of tumor angiogenesis by metformin treatment via a mechanism linked to targeting of HER2/ HIF-1 $\alpha /$ VEGF secretion axis. Oncotarget. 2015;6(42):44579-44592.

22. Li LJ, Huang Q, Zhang N, Wang GB, Liu YH. miR-376b-5p regulates angiogenesis in cerebral ischemia. Mol Med Rep. 2014;10(1):527-535.

23. Rane JK, Scaravilli M, Ylipää A, et al. MicroRNA expression profile of primary prostate cancer stem cells as a source of biomarkers and therapeutic targets. Eur Urol. 2015;67(1):7-10.

24. Lu J, Zhang M, Yang X, Cui T, Dai J. MicroRNA-548c-3p inhibits T98G glioma cell proliferation and migration by downregulating c-Myb. Oncol Lett. 2017;13(5):3866-3872.

25. Chang H, Kim N, Park JH, et al. Different microRNA expression levels in gastric cancer depending on Helicobacter pylori infection. Gut Liver. 2015;9(2):188-196.

26. Tormo E, Pineda B, Serna E, et al. MicroRNA profile in response to doxorubicin treatment in breast cancer. J Cell Biochem. 2015;116(9):2061-2073.
27. McGuire A, Brown JA, Malone C, McLaughlin R, Kerin MJ. Effects of age on the detection and management of breast cancer. Cancers. 2015; 7(2):908-929.

28. Zahmatkesh B, Keramat A, Alavi N, et al. Breast cancer trend in Iran from 2000 to 2009 and prediction till 2020 using a trend analysis method. Asian Pac J Cancer Prev. 2016;17(3):1493-1498.

29. Szakács G, Paterson JK, Ludwig JA, Booth-Genthe C, Gottesman MM. Targeting multidrug resistance in cancer. Nat Rev Drug Discov. 2006; 5(3):219-234.

30. Kars MD, Işeri OD, Gunduz U, Molnar J. Reversal of multidrug resistance by synthetic and natural compounds in drug-resistant MCF-7 cell lines. Chemotherapy. 2008;54(3):194-200.

31. Yang YX, Wei L, Zhang YJ, et al. Long non-coding RNA p10247, high expressed in breast cancer (lncRNA-BCHE), is correlated with metastasis. Clin Exp Metastasis. 2018;35(3):109-121.

32. Song R, Zhang J, Huang J, Hai T, Huang HT. Long non-coding RNA GHET1 promotes human breast cancer cell proliferation, invasion and migration via affecting epithelial mesenchymal transition. Cancer Biomark. 2018;22(3):565-573.

33. Luo Z, Li D, Luo X, et al. Decreased expression of miR-548c-3p in osteosarcoma contributes to cell proliferation via targeting ITGAV. Cancer Biother Radiopharm. 2016;31(5):153-158.

34. Wang JC, Li XX, Sun X, et al. Activation of AMPK by simvastatin inhibited breast tumor angiogenesis via impeding HIF-1 $\alpha$-induced pro-angiogenic factor. Cancer Sci. 2018;109(5):1627-1637.

35. Ni X, Zhao Y, Ma J, et al. Hypoxia-induced factor-1 alpha upregulates vascular endothelial growth factor $\mathrm{C}$ to promote lymphangiogenesis and angiogenesis in breast cancer patients. J Biomed Res. 2013;27(6): 478-485.

36. Kim A, Ma JY. Rhaponticin decreases the metastatic and angiogenic abilities of cancer cells via suppression of the HIF-1 $\alpha$ pathway. Int $J$ Oncol. 2018;53(3):1160-1170.

37. Nan Y, Guo H, Guo L, et al. miRNA-451 inhibits glioma cell proliferation and invasion through the mTOR/HIF-1 $\alpha /$ VEGF signaling pathway by targeting Cab39. Hum Gene Ther Clin Dev. 2018;29(3): 156-166.

38. Zhu H, Zhang S. Hypoxia inducible factor- $1 \alpha /$ vascular endothelial growth factor signaling activation correlates with response to radiotherapy and its inhibition reduces hypoxia-induced angiogenesis in lung cancer. J Cell Biochem. 2018;119(9):7707-7718.
OncoTargets and Therapy

\section{Publish your work in this journal}

OncoTargets and Therapy is an international, peer-reviewed, open access journal focusing on the pathological basis of all cancers, potential targets for therapy and treatment protocols employed to improve the management of cancer patients. The journal also focuses on the impact of management programs and new therapeutic agents and protocols on

\section{Dovepress}

patient perspectives such as quality of life, adherence and satisfaction The manuscript management system is completely online and includes a very quick and fair peer-review system, which is all easy to use. Visit http://www.dovepress.com/testimonials.php to read real quotes from published authors. 